\title{
Genetic and environmental contributions to variation in human tooth size
}

\author{
P. J. DEMPSEY† \& G. C. TOWNSEND* + \\ $\dagger$ South Australian Centre for Rural and Remote Health, University of South Australia, Nicolson Ave., Whyalla \\ Norrie, 5608, Australia and $\ddagger$ Dental School, University of Adelaide, Adelaide, 5005, Australia
}

\begin{abstract}
Human dental crowns are complex structures without simple genetic or environmental determination, but mathematical modelling of data from family studies is now providing a more complete picture of their ontogeny. Mesiodistal (MD) and buccolingual (BL) dental crown diameters were recorded from almost 600 monozygotic and dizygotic twins, and univariate biometrical models were fitted to the data for 28 permanent teeth (excluding third molars). All 56 variables showed significant contributions of additive genetic variation, varying from 56 to $92 \%$ of phenotypic variation, with most being over $80 \%$. The effects of individual or unique environment ranged from 8 to $29 \%$. A significant effect of the environment shared by twins - either uterine or early childhood - was found for MD and BL diameters of maxillary first molars (22-27\%). There were also significant levels of non-additive genetic variation in MD diameters of canines and first premolars, which is consistent with selective pressures acting on these teeth at some stage in human evolution.
\end{abstract}

Keywords: dentition, genetic modelling, tooth size, twins.

\section{Introduction}

Two main quantitative genetic approaches have been used previously by researchers seeking to clarify the causes of observed variation in the human dentition: classical correlation analysis and multiple abstract variance analysis (MAVA). The classical correlation approach uses correlations between relatives to estimate various ratios that quantify the relative importance of genetic and environmental influences on trait variation (Jinks \& Fulker, 1970). Such parameters include the $H$ of Holzinger (1929), $E$ of Neel \& Schull (1954) and $H R$ of Nichols (1965)), but the best-known example is probably the heritability estimate, $h^{2}=2\left(r_{\mathrm{mz}}-r_{\mathrm{dz}}\right)$ described by Bulmer (1970) and Smith (1974, 1975). MAVA is a more systematic and comprehensive approach that compares within- and between-family variances for twins, full-sibs and half-sibs. It leads to the estimation of nature:nurture ratios, and assesses correlations between genetic and environmental influences within the family and within the culture (Jinks \& Fulker, 1970).

Published estimates of heritability for dental crown size in human populations vary, although most are over $60 \%$. (Harzer, 1987; Townsend, 1992). However, previous studies have been limited in the information that

*Correspondence. E-mail: grant.townsend@adelaide.edu.au could be obtained from the data, and they also were based on unlikely assumptions. For instance, a common assumption was that family environment (also called shared or common environment) had no impact on the traits under study.

A third quantitative genetic method, Fisher's biometrical approach, is considered to have several advantages over both correlation analysis and MAVA (Jinks \& Fulker, 1970). It represents a major breakthrough in estimating various genetic and environmental effects, since it allows testing for, and estimation of, the components that were previously assumed to be absent or undetectable. The advantages are considered significant enough to justify a new analysis of human dental crown size for genetic and environmental sources of variation. In particular, the relative role of common or shared environment may be estimated and genotypeenvironment interactions tested.

Butler's (1939) field concept states that tooth primordia grow and differentiate within 'fields' of diffusing morphogenetic substances, with one field for each tooth type. A tooth growing in the centre of a field (known as the polar or key tooth), should show less variation than teeth on either side, and the further from the centre, the more variable the teeth should be. In humans, four morphogenetic fields have been identified - incisor, canine, premolar, molar (Butler, 1939; Dahlberg, 1945). 
Within these fields, the upper central incisor, lower lateral incisor, canine, first premolar and first molar have been identified as the polar teeth.

Genetic theory holds that traits with low heritability tend to be strongly associated with reproductive fitness (Falconer, 1989). These traits also exhibit more stability with time, and tend to be less variable in overall morphology. Thus, polar teeth might be expected to show lower levels of both variation and heritability than other members of the same tooth group. Evidence for Butler's morphogenetic fields has been sought in the past by comparing heritability estimates. The findings have been inconsistent, with some studies revealing lower heritabilities in polar teeth as predicted (Alvesalo \& Tigerstedt, 1974; Potter et al., 1978), and others revealing a different pattern or apparent lack of pattern (Harzer, 1987). This inconsistency may be evidence against the concept, or it may be due to differences in statistical methods in the population from which the sample originated, or due to random variation.

The aims of this study were to quantify the relative contributions of various genetic and environmental factors to variation in $\mathrm{MD}$ and $\mathrm{BL}$ dimensions of permanent tooth crowns; and to examine patterns of heritabilities for agreement with previous findings, particularly, with Butler's field concept.

\section{Materials and methods}

\section{Data collection}

The data used for this study were collected from identical (monozygotic or MZ) and nonidentical (dizygotic or DZ) twins. All subjects were of European descent. Data were obtained from 596 subjects, incorporating $149 \mathrm{MZ}$ twin pairs (83 female, 66 male) and 149 DZ twin pairs (49 female, 44 male, 56 femalemale). Throughout this paper, female-male twin pairs are referred to as opposite-sex (OS) twins, contrasting with (female-female or male-male) same-sex (SS) twins. The twins were aged from 7 to 62 years, with $90 \%$ between 10 and 25 years. The mean age was 16.5 years. Data collection methods were approved by the Committee on the Ethics of Human Experimentation, University of Adelaide (Approval No. H/07/84) and all participants were informed volunteers. Detailed methods of zygosity determination have been reported elsewhere (Dempsey et al., 1995). In brief, the probability of dizygosity given concordance for all blood systems was less than $1 \%$.

For each subject, alginate impressions of maxillary and mandibular dental arches were obtained, from which stone models were cast and measured. Maximum

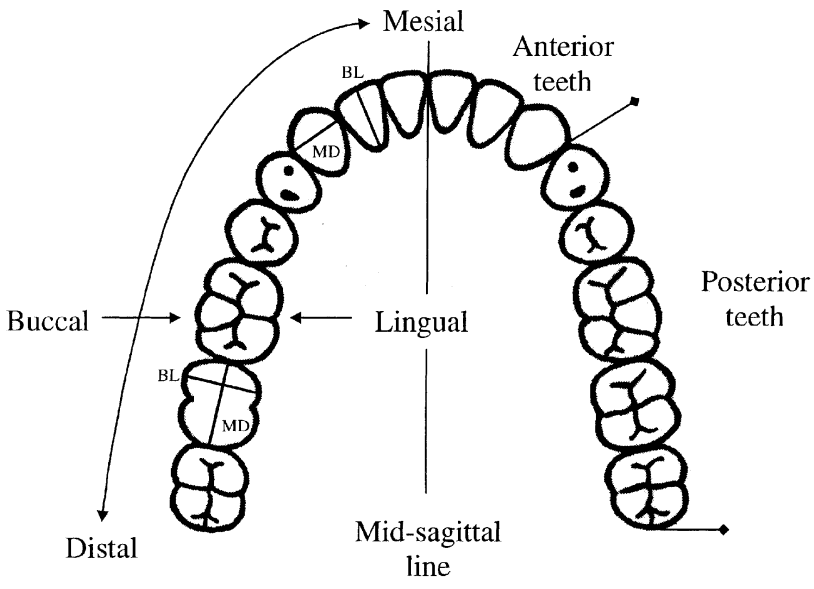

Fig. 1 Dental nomenclature. MD, mesiodistal diameter; BL, buccolingual diameter.

mesiodistal (MD) and buccolingual (BL) crown diameters were recorded, following the definitions of Moorrees et al. (1957), from all emerged and sufficiently intact permanent teeth. Anatomically, the mesiodistal diameter is the length of the tooth, and BL diameter is the breadth. However, the MD diameter is commonly thought of as the tooth width, and the BL diameter as the thickness through the tooth (Fig. 1). Third molars were excluded from the study, yielding a maximum of 56 variables per subject. The measuring equipment and estimates of reliability of the measurement technique have been reported by Dempsey et al. (1995), with errors of the method being small and unlikely to bias the results.

Corrections for age were not considered necessary since the final size of dental crowns is determined before emergence of the teeth into the oral cavity, and any teeth displaying significant wear at measurement sites were excluded from subsequent analyses.

\section{Preliminary analyses}

Given that multiple tests were carried out on the inter-correlated dental variables, both in the preliminary analyses and in the genetic model fitting, alpha $(\alpha)$ levels were chosen for rejection of null hypotheses following the Bonferroni inequality (Hair et al., 1995).

Initial analyses of the data to test for normality were performed for males and females separately. Kolmogorov-Smirnov test statistics, probability plots and estimates of skewness and kurtosis, indicated that the tooth size data were normally distributed.

Correlation analyses performed between MD and BL diameters within teeth yielded coefficients that were all positive, ranging in value from 0.35 to 0.74 . 
The values of correlation coefficients calculated between teeth ranged from 0.27 to 0.93 for $\mathrm{MD}$ dimensions, and from 0.41 to 0.93 for BL dimensions. Correlations were highest for antimeric teeth or for adjacent teeth within the same tooth class, i.e. incisors, premolars or molars, whereas correlations between other variables were moderate to low.

\section{Partitioning phenotypic variation}

The twin method may be applied to quantitative data to estimate the relative influences of additive genetic factors (A), non-additive genetic factors - dominance and epistatic interactions between loci (D), common or shared environment $(\mathrm{C})$ and that part of the environment which is unique to each individual (E). This involves comparing $\mathrm{MZ}$ twin variances and covariances with those of DZ twins. More detail on this process is contained in Neale \& Cardon (1992) and Dempsey et al. (1995).

Before attempting to estimate these four factors, the contribution of three additional sources of variance and covariance should be considered - assortative mating, genotype-by-environment interaction $(\mathrm{G} \times \mathrm{E})$ and genotype-environment correlation (CorGE). The influences of these factors are described in detail elsewhere (Neale \& Cardon, 1992), so each will only be described briefly.

Assortative mating mimics common environment, spuriously lowering the genetic contribution to variation. In order to test for its presence, data from spouses, or from parents of twins, are required. Since the evidence published to date suggests that there is no assortative mating with respect to tooth crown size in humans (Townsend \& Brown, 1978; El-Nofely \& Tawfik, 1995), assortative mating was assumed to be absent.

Genotype-by-environment $(\mathrm{G} \times \mathrm{E})$ interaction describes the situation in which one genotype may be expressed in the same way in two different environments, whereas another genotype changes. To test for $\mathrm{G} \times \mathrm{E}$ interactions, regression analysis of $\mathrm{MZ}$ pair variances on $\mathrm{MZ}$ pair means was performed for each variable (Jinks \& Fulker, 1970).

Genotype-environment correlation (CorGE) occurs when the environments an individual experiences are not a random sample of all environments, but are influenced by, or correlated with, the individual's genotype. There are several types, each with a different effect. Genetic variance may be increased or decreased, and variances between MZ and DZ twins, or between twins and singletons, may be unequal. CorGE includes sibling effects, wherein the phenotype of one sibling influences the phenotype of another. For example, sibling competition may arise if a twin transfusion effect operated in utero. This would lead to a decrease in the estimated amount of genetic variance.

Unfortunately, CorGE cannot be detected in crosssectional studies like this one, requiring adoption data and/or a variety of different familial relationships (Neale \& Cardon, 1992), so it must be assumed to have little or no impact. There is some reassurance in noting that twin transfusion effect, and other sources of sibling interaction, have not been shown to influence tooth crown size substantially in twins. In fact, very few examples of CorGE have been demonstrated in humans. After 20 or more years of testing for CorGE using well-designed, powerful empirical studies (Martin et al., 1997), the assumption that it is not significant seems realistic.

\section{Testing for $G \times E$ interaction}

Before proceeding with modelling of covariance structure, the data were explored to test for the presence of genotype-environment $(\mathrm{G} \times \mathrm{E})$ interactions. Significant regression of $\mathrm{MZ}$ pair variances on $\mathrm{MZ}$ pair means provides an indication of $\mathrm{G} \times \mathrm{E}$ interaction (Jinks \& Fulker, 1970). To this end, the absolute MZ pair difference (which is proportional to the square root of the intra-pair variance) was regressed onto MZ pair sum, and also onto the square of the pair sum. In case the relationship was not linear, square and logarithmic (log) transformations of the data were tested also for significant regression. In total, 224 regressions were calculated and significance levels were adjusted for multiple comparisons.

\section{Modelling of means}

The Mx program developed by Neale (1995) permits modelling of means, variances and covariances simultaneously. To simplify the process, a variety of mean models may be fitted while the variance-covariance matrix is held constant. Modelling of the latter can then proceed, incorporating the best mean model. For each of the 56 variables, five models of the means were fitted, each model building on the preceding one. In the first model, all twins were constrained to have the same mean. Female and male twins were allowed different means in the next model. For the third model, females from OS twin pairs were allowed to vary from SS female pairs. The fourth model permitted the same for males as well. The final model removed all constraints of equality, except between co-twins of same-sex pairs, yielding six separate means.

Initially, the simplest model that would explain the data was sought. Given the number of $\chi^{2}$ tests performed, alpha $(\alpha)$ levels were adjusted for multiple 
testing before testing. Secondly, more complicated models were checked to see if any provided a significantly better fit.

\section{Modelling of variances and covariances}

Once the appropriate mean model was found, models were fitted to covariances. Each variable was analysed separately, fitting a path coefficient model with unique environmental influences only ( $\mathrm{E}$ model). Where $\chi^{2}$ analysis indicated that the $\mathrm{E}$ model did not provide a good fit to the data, the model was extended to include additive genetic variation (AE model), and then common environmental variation (CE model). Finally, ACE and ADE models were fitted, where D (non-additive genetic variation) incorporates both dominance and epistatic interaction variance, which cannot be separated when only MZ and DZ twins are used (Mather, 1974). Path coefficients $(a, c, d, e)$ were estimated, and $\chi^{2}$ values for goodness-of-fit of the models were calculated. Akaike's Information Criterion (AIC $=\chi^{2}$ minus two times the degrees of freedom) was used to indicate goodness of fit penalized for model complexity (Akaike, 1987). The smaller or more negative the AIC, the better the parsimony and fit of a model. Various hypotheses were tested by setting different combinations of paths to zero, and examining the difference between resulting $\chi^{2}$ or AIC values. Again, the general approach was that of only accepting a more complex model when a simpler one did not provide a good fit to the data, or when the more complex model was significantly better by $\chi^{2}$ $(\alpha=0.05)$. These $\chi^{2}$ values between two models are denoted $\chi_{\text {diff. }}^{2}$

Comparisons of $\chi^{2}$ and AIC values between complex and simpler models also allow calculation of the significance of path coefficients $(\alpha=0.05)$. Only $e$ could not be tested for its significance, as no model excluded it. In general, it is deemed nonsensical to exclude $\mathrm{E}$ from models, since it incorporates measurement error. In addition, correlations between MZ twins were less than 1.00 , indicating the presence of unique environmental variation.

More complex models were then applied to those variables without a satisfactory model in the first phase. Firstly, OS twins were removed from the analysis, then male and female SS twin pairs were analysed separately. Heterogeneity of the sexes was assessed by likelihood ratio $\chi^{2}(\alpha=0.05)$, subtracting the sum of the $\chi^{2}$ values for each sex from the $\chi^{2}$ for the four SS twin groups. The resulting value itself is distributed as $\chi^{2}: \chi^{2}=\chi_{\mathrm{m} \& \mathrm{f}}^{2}$ $\left[\chi_{\mathrm{m}}^{2}+\chi_{\mathrm{f}}^{2}\right]$ with degrees of freedom (d.f.) $=$ d.f. .m\&f $^{-}$ [d.f.m + d.f.f $]$.

A variety of sex-limitation models can be applied also, so the variances, covariances and co-twin correlations were examined first to see which model(s) were likely. Possible models included heterogeneity, scalar sex-limitation, non-scalar sex-limitation, and general sex-limitation models. A more detailed description of these models is contained in Neale \& Cardon (1992).

Once a model had been chosen for each variable, heritability $\left(h^{2}\right)$ was estimated from the ratio of genetic variation to total phenotypic variation, using parameter estimates from the model chosen as the best using the above criteria.

\section{Results}

\section{$G \times E$ interaction}

There was no evidence of $\mathrm{G} \times \mathrm{E}$ interaction in any of the 56 variables, since none of the 224 regressions were statistically significant.

\section{Modelling of means}

The simplest model — one mean for all twins — was sufficient for only 10 of the 56 variables. For reasons of consistency between covariance models, it was therefore decided to proceed with modelling the covariances using a mean model with a different parameter for the mean of each sex.

\section{Modelling of variances and covariances}

In the covariance modelling phase, models with only a unique environmental factor (E) were rejected $(P<0.001)$ for all variables. Adding shared environment (C) did not result in an adequate fit, except for three maxillary variables - the right second premolar MD diameter, and both dimensions of the left second molar. For all three variables, the AE model gave a substantially better fit by AIC. In fact, the AE model was adequate for all but two of the 56 variables.

For eight of the 56 variables, an additional factor (either common environmental variation or non-additive genetic variation) was found to be significant. Some improvement in fit was observed when common environment was included (ACE model) for BL and MD dimensions of maxillary right and left first molars. This was significant for the maxillary left variables, and almost so for the right side $(0.05<P<0.10)$. Likewise, addition of non-additive genetic variation (ADE model) resulted in improvement in fit for MD diameters of the four canines, both maxillary first premolars, and mandibular right first premolar, as well as the BL diameter of the maxillary right first premolar. The improvement was significant for all but the mandibular right canine and maxillary right first premolar $\mathrm{MD}$, 

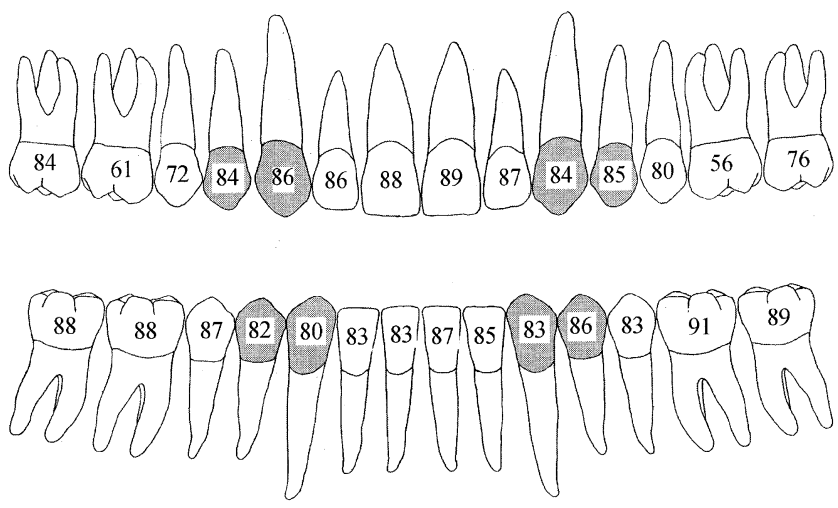

Fig. 2 Heritability of MD diameter. Unshaded teeth, additive genetic variance (narrow heritability); shaded teeth, additive + non-additive genetic variance (broad heritability).
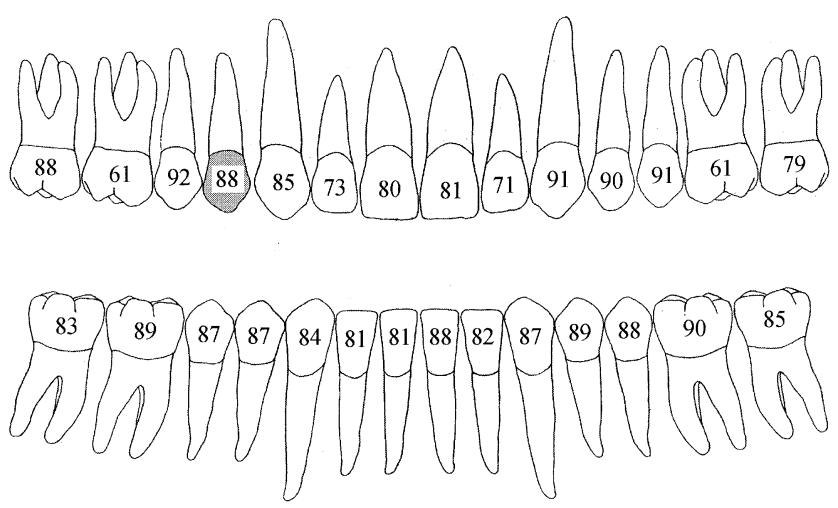

Fig. 3 Heritability of BL diameter. Unshaded teeth, additive genetic variance (narrow heritability); shaded teeth, additive + non-additive genetic variance (broad heritability).

although these almost attained significance $(0.05<P<0.10)$.

Standardized parameter estimates for each variable, calculated by dividing the specific variance components by the phenotypic variance, are depicted in Figs $2-5$.

\section{Heterogeneity between sexes}

Two exceptions for which none of the models provided a statistically good fit were the BL diameters of the maxillary left central incisor and right canine. The AE model was sufficient for their antimeres, with probabilities of $0.02<P<0.05$ and $0.05<P<0.10$, respectively.

Excluding the OS twins did not improve the situation, but separate analyses for each sex resulted in considerably improved model fitting. For the BL dimension of the maxillary left central incisor, the $\mathrm{AE}$
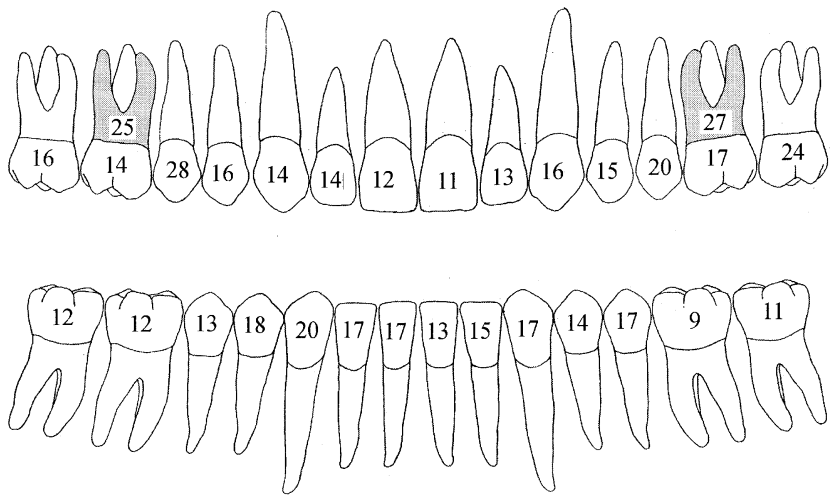

Fig. 4 Environmentality of MD diameter. Unshaded region, unique environmental variance; shaded region, common environmental variance.
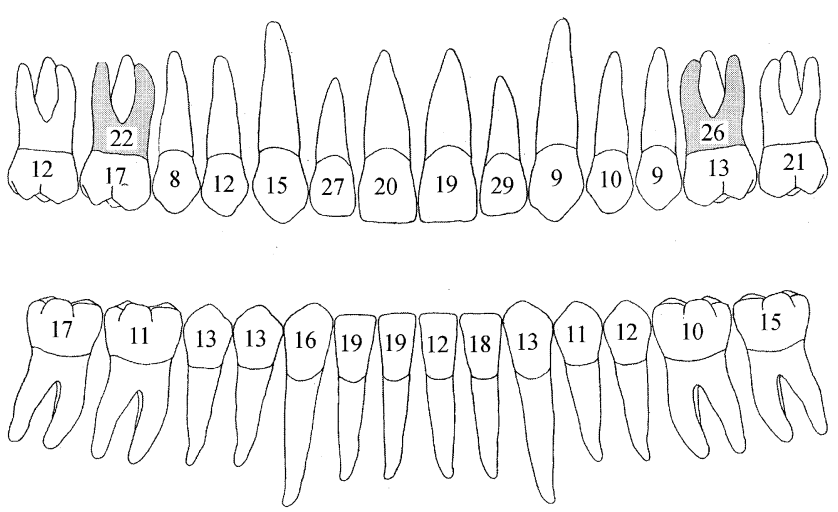

Fig. 5 Environmentality of BL diameter. Unshaded region, unique environmental variance; shaded region, common environmental variance.

model was best for both sexes, and $a$ was the only significant parameter. For the antimere, the AE model was best in females whereas the ACE model was best in males, although again $a$ was the only significant parameter. For both maxillary canine BL breadths, the ACE model was best for females and AE for males. Significant parameters were $a$ and $c$ for females, and $a$ for the left canine in males. Tests of heterogeneity between sexes were significant for the two variables and their antimeres, whether using AE or ACE models. This suggests that sex-limited differences may exist in one or more of the parameters.

In the next stage, models were fitted which attempted to encompass sex-limited gene or environmental factors. Examination of the variances and co-twin correlations revealed little about the likely type of sex-limited effects which might have occurred. Thus, all types of models were fitted - heterogeneity, scalar

(C) The Genetics Society of Great Britain, Heredity, 86, 685-693. 
sex limitation, nonscalar sex limitation and general sex limitation.

For both central incisors, the best model was a scalar sex-limitation AE model. Estimated values for $k$, the scalar by which male statistics differed from those of females, was 1.23 for the left and 1.16 for the right central incisor.

The models for canines were less consistent. The best model for the maxillary right canine BL diameter was a general sex-limitation ACE model with separate A, C and $\mathrm{E}$ parameters for each sex. For the antimere, a scalar sex-limitation ACE model was the best, with estimated $k=1.19$.

\section{Parameter estimates}

The parameter estimates derived from the best model (squared, standardized and $\times 100$ ) appear in Figs 3-5. For variables with some influence of non-additive genetic variation, estimates for $\mathrm{A}$ and $\mathrm{D}$ were confounded, so separate values could not be derived, only total genetic contribution. In these variables, the heritability estimate is actually the ratio of total genetic to phenotypic variation (broad heritability).

Among the variables for which the $\mathrm{AE}$ or $\mathrm{ADE}$ models were chosen, heritability estimates ranged from 71 to $92 \%$ (most were over $80 \%$ ), with the remaining $8-29 \%$ being the unique environment contribution. In the ACE models of the other variables - MD and BL diameters of maxillary first molars - additive genetic variation accounted for $56-61 \%$ of the variation, shared environment for $22-27 \%$, and unique environment, $12-17 \%$. Although additive genetic variation contributed most, there were substantial common environmental contributions to variation in both dimensions of the maxillary first molars.

\section{Discussion}

In interpreting the findings of the present analysis, it is important to bear in mind that the sizes of different tooth crowns within the oral cavity are inter-correlated. Correlations between antimeric and adjacent teeth are reasonably high, whereas those between more distant teeth are only moderate to low in magnitude. The two measures of crown size employed in this study, MD and BL diameters, are also correlated.

Although a full multivariate analysis would have overcome many of the limitations of our univariate approach, it was not possible due to constraints within the Mx program and in available computer memory. Nevertheless, our previous multivariate analyses of groups of teeth, including maxillary and mandibular incisors, or teeth in a single quadrant, have yielded similar estimates of genetic and environmental contributions to observed variation as those obtained in the present study (Dempsey et al., 1995; Dempsey et al., 1999). In addition, the present analysis enabled us to examine patterns of narrow-sense heritabilities between the permanent teeth and compare findings with those of previous researchers.

\section{Univariate analyses of means}

The finding that most variables were best modelled with two means, one for each sex, is consistent with frequent reports of significant sexual dimorphism for permanent tooth size (Alvesalo, 1971; Hanihara, 1978; Oxnard, 1987). The mandibular lateral incisors, often amongst the least sexually dimorphic permanent teeth, did not require separate means for each sex.

\section{Univariate analyses of covariance structure}

The main finding from the univariate analyses was that variation in crown size of most teeth was explicable by additive genetic and unique environmental variation, with no need for non-additive genetic or shared environmental variation. Most previous analyses of genetic structure of tooth crown size have yielded the same conclusion (Potter et al., 1983). There was also a high degree of bilateral symmetry in the parameter estimates, with a maximum difference of $9 \%$ between antimeres.

\section{Common environmental variation}

The first exception to the 'AE model rule' involved the indication of common environmental variation in $\mathrm{MD}$ and BL dimensions of the maxillary first molars. Although significance was only achieved for both diameters of the left first molar, the probability values for the right side were close to significant $(0.05<P<0.10)$, suggesting that common environmental variation may have been contributing also to the size of this tooth. This contrasts with a prior analysis of genetic structure, involving MD diameters of maxillary first molars, in which no evidence was found for common environmental variation (Potter et al., 1983).

The first molar is exceptional among permanent teeth because it begins to calcify at birth, or soon after. The soft-tissue phase, during which a tooth's form is still malleable, ends with completion of calcification. If any aspect of the uterine environment affects tooth crown size, it might be expected to manifest itself as a common environmental factor, and most likely would be expressed in the deciduous teeth and permanent first molars. The presence of significant common environ- 
ment in this tooth therefore could be taken as indirect evidence for a prenatal, or early postnatal, common environmental influence contributing between 22 and $27 \%$ of the total variation in maxillary first molar crown size.

These estimates are similar in magnitude to those from a study of indigenous Australian families (Townsend, 1992), although a general common environmental factor was postulated in that case. Common environmental contributions to the permanent teeth were estimated to average $18 \%$ for $\mathrm{BL}$ and $10 \%$ for MD diameters. A further pattern reported by Townsend was of higher values for BL than MD diameters, but there was no evidence of such a pattern in the current study.

\section{Non-additive genetic variation}

The second exception to the AE model rule concerned the presence of non-additive genetic variation in the canines and first premolars. This affected the MD diameters almost exclusively. The single BL diameter, that of the right maxillary first premolar, may reflect the presence of a low degree of non-additive genetic variation in BL diameters of first premolars, or it may be an artefact of the correlation that exists between MD and $\mathrm{BL}$ diameters.

Genes that are related to selective fitness tend to display non-additive genetic variation (Fisher, 1958; Kacser \& Burns, 1981; Dean et al., 1988), so the presence of this type of variation may indicate selective pressures acting either currently or sometime in the past. Canines and first premolars are 'linked' in their occlusal functioning within the dentition, and possibly also in their evolution. Maxillary canines are larger and considerably stronger than other teeth, so the presence of strong first premolars and mandibular canines is likely to have provided some selective advantage.

\section{Sexual dimorphism}

There was significant sexual dimorphism for tooth crown size, since the mean tooth sizes of the twins could be constrained to be equal across zygosities within each sex, but not across sexes. This dimorphism did not extend to covariance structure, except for BL breadths of the maxillary left central incisor and right canine, which required heterogeneous models for the sexes.

For maxillary central incisors, the best model was a scalar sex-limitation AE model. In this model, estimates for $\mathrm{A}, \mathrm{C}$ and $\mathrm{E}$ were the same for the two sexes, except that male estimates were allowed to vary from female ones by a multiple, $k$. The value of $k$ averaged over the antimeres was 1.2, compared with the difference in means between sexes of 1.04. Given the probabilities associated with these models based on $\chi^{2}$ tests of goodness of fit $(0.05<P<0.10$ for left, and $0.10<P<0.20$ for right, central incisors), they should be viewed with caution. A number of other models also yielded similar goodness-of-fit, with the best model being chosen by AIC.

A similar situation exists for the BL diameter of maxillary canines. Although higher probabilities were achieved for canines $(P>0.30$ for right, $P>0.20$ for left) than for incisors, the best models for each antimere were quite inconsistent. For the maxillary right canine, the best models were general sex-limitation models, with two or more parameters having separate estimates in each of the sexes. For the antimere, scalar ACE and AE models were the best (estimated $k=1.19$ ).

Caution is therefore warranted in the extent to which these sex-limitation models are invoked in future discussions of dental genetics. It is possible that the models specified did not take into account some important factor, or that the data themselves were somehow problematic. The only way to resolve this would be to collect a second sample and repeat the modelling.

\section{Heritability estimates and Butler's field concept}

The heritability estimates were moderate to high, with a minimum of $50-60 \%$ for the MD length of the maxillary first molar, reaching up to around $90 \%$ for the BL breadth of the maxillary premolars. These estimates extend across most of the range of previously reported heritabilities. Shared environment contributed up to $27 \%$, and unique environment, $29 \%$, indicating that environmental influences on tooth crown size can be substantial.

Studies of twins reared apart provide a powerful means of estimating heritabilities by overcoming some of the confounding effects of environmental influences shared by twins living together. Boraas et al. (1988) took advantage of this approach to demonstrate a significant genetic contribution to permanent incisor tooth size, intraclass correlations in $\mathrm{MZ}$ twins reared apart (which provide a direct measure of heritability) being 0.94 for combined maxillary incisor size and 0.63 for combined mandibular size. These findings for incisors are therefore consistent with our estimates based on $\mathrm{MZ}$ twins reared together.

There was no consistent pattern to the heritabilities, either comparing mesial and distal members of tooth groups, or MD and BL dimensions of individual teeth. Of the 24 mesial: distal comparisons that could be made, 11 were in the predicted direction of mesial $>$ distal (incorporating reversal for the mandibular incisors). Of 
the $28 \mathrm{MD}$ : BL comparisons, 13 were in the direction $\mathrm{MD}>\mathrm{BL}$.

\section{Conclusions}

Our results show that additive genetic effects contribute significantly to variation in human permanent dental crown size. Significant levels of non-additive genetic variance were noted for mesiodistal dimensions of canines and first premolars, suggesting that selective pressures may have acted specifically on these teeth during the course of human evolution. A significant shared or common environmental influence on maxillary first molar crown size is consistent with their early development, calcification commencing around birth. Our results provide no evidence of systematic differences in heritabilities for crown size between different teeth within each tooth class, as expected under Butler's morphogenetic field concept, or between different crown dimensions of permanent teeth. Multivariate genetic analyses that take account of the intercorrelations in measures of crown size within and between teeth are now planned to further clarify the role of genetic and environmental influences on the human dentition.

\section{Acknowledgements}

This research was supported by a project grant from the National Health and Medical Research Council of Australia. The support of the Australian Twin Registry is gratefully acknowledged, as is the participation of the twins. Professors Nick Martin (Queensland Institute of Medical Research) and Mike Neale (Medical College of Virginia) provided helpful comments during data collection and/or analysis.

\section{References}

AKAIKe, H. 1987. Factor analysis and AIC. Psychometrika, 52, 317-332.

ALVESAlO, L. 1971. The influence of sex-chromosome genes on tooth size in man. A genetic and quantitative study. Proc. Finn. Dent. Soc., 67, 3-54.

Alvesalo, L. AND TIGERSTEDT, P. M. A. 1974. Heritabilities of human tooth dimensions. Hereditas, 77, 311-318.

BORAAS, J. C., MESSER, L. B. AND TILL, M. J. 1988. A genetic contribution to dental caries, occlusion, and morphology as demonstrated by twins reared apart. J. Dent. Res., 67, 1150-1155.

Bulmer, M. G. 1970. The Biology of Twinning in Man. Clarendon, Oxford.

BUTLER, P. M. 1939. Studies of the mammalian dentition differentiation of the post-canine dentition. Proc. Zool. Soc. Lond., Ser. B., 109, 1-36.
Dahlberg, A. A. 1945 . The changing dentition of man. J. Am. Dent. Ass., 32, 676-690.

DEAN, A. M., DYKHUIZEN, D. E. AND HARTL, D. L. 1988. Theories of metabolic control in quantitative genetics. In: Proceedings of the Second International Conference on Quantitative Genetics, pp. 536-548. Sinauer, Sunderland, MA.

DEMPSEY, P. J., TOWNSEND, G. C., MARTIN, N. G. AND NEALE, M. C. 1995. Genetic covariance structure of incisor crown size in twins. J. Dent. Res., 74, 1389-1398.

DEMPSEY, P. J., TOWNSEND, G. C. AND MARTIN, N. G. 1999. Insights into the genetic basis of human dental variation from statistical modelling analyses. Perspect. Hum. Biol., 4, 9-17.

EL-NOFELY, A. AND TAWFIK, W. A. 1995. On inheritance of permanent tooth crown size in a Middle Eastern population. Homo, 46, 51-62.

FALCONER, D. S. 1989. Introduction to Quantitative Genetics, 3rd edn. Longman Scientific \& Technical, Essex, England.

FISHER, R. A. 1958. The Genetical Theory of Natural Selection, 2nd edn. Dover Publications, New York.

HAIR, J. F., ANDERSON, R. E., TATHAN, R. L. AND BLACK, W. C. 1995. Multivariate Data Analysis with Readings, 4th edn, p. 281. Prentice Hall, NJ.

HANiHARA, K. 1978. Differences in sexual dimorphism in dental morphology among several human populations. In: Butler, P. M. and Joysey, K. A. (eds) Development, Function and Evolution of Teeth, pp. 127-133. Academic Press, London.

HARZER, w. 1987. A hypothetical model of genetic control of tooth-crown growth in man. Arch. Oral Biol., 32, 159-162.

HOLZINGER, K. J. 1929. The relative effect of nature and nurture influences on twin differences. J. Educ. Psychol., 20, 245-248.

JINKS, J. L. AND FUlKeR, D. W. 1970. Comparison of the biometrical genetical, MAVA, and classical approaches to the analysis of human behavior. Psychol. Bull., 73, 311-348.

KACSER, H. AND BURNS, J. A. 1981. The molecular basis of dominance. Genetics, 97, 639-674.

MARTIN, N., BOOMSMA, D. AND MACHIN, G. 1997. A twinpronged attack on complex traits. Nature. Genet., 17, 387-392.

MATHER, K. 1974. Non-allelic interaction in continuous variation of randomly breeding populations. Heredity, 32, 414-419.

MOORREES, C. F. A., THOMSEN, S. O., JENSEN, E. AND YEN, P. K. J. 1957. Mesiodistal crown diameters of the deciduous and permanent teeth in individuals. J. Dent. Res., 36, 39-47.

NEALE, M. C. 1995. Mx: Statistical Modeling, 3rd edn. Department of Psychiatry, Virginia Commonwealth University.

NEALE, M. C. AND CARDON, L. R. 1992. Methodology for Genetic Studies of Twins and Families. Kluwer Academic Publishers, Dordrecht, The Netherlands.

NEEl, J. V. AND SCHull, w. J. 1954. Human Heredity. University of Chicago Press, Chicago.

NICHOLS, R. C. 1965. The national merit twin study. In: Vandenberg, S. G. (ed.) Methods and Goals in Human Behavior Genetics, pp. 231-243. Academic Press, New York. oxnARD, C. E. 1987. Fossils, Teeth and Sex. New Perspectives on Human Evolution. Hong Kong University Press, Hong Kong. 
POTTER, R. H. Y., NANCE, W. E. AND YU, P. L. 1978. Genetic determinants of dental dimensions: a twin study. Prog. Clin. Biol. Res., 24, 235-240.

POTTER, R. H. Y., RICE, J. P., DAHLBERG, A. A. AND DAHLBERG, T. 1983. Dental size traits within families: path analysis for first molar and lateral incisor. Am. J. Phys. Anthropol., 61, 283-289.

SMITH, C. 1974. Concordance in twins: methods and interpretation. Am. J. Hum. Genet., 26, 454-466.
SMITH, C. 1975. Quantitative inheritance. In: Textbook of Human Genetics, pp. 382-441. Blackwell, Oxford.

TOWNSEND, G. C. 1992. Genetic and environmental contributions to morphometric dental variation. J. Hum. Ecol., Special Issue, 2, 61-72.

TOWNSEND, G. C. AND BROWN, T. 1978. Inheritance of tooth size in Australian Aborigines. Am. J. Phys. Anthropol., 48, 305-314. 